\title{
Comportamento da Variabilidade da Frequência Cardíaca em Teste com Cargas Progressivas
}

\author{
Proceeding of the Cardiac Frequency Variability in Test with Progressive \\ Loads
}

\begin{abstract}
Alan Peloso Figueiredo ${ }^{1,5}$; Bianca Martins de Figueiredo ${ }^{2}$; Giuliano Roberto da Silva $^{3,4}$; José Carlos Ferreira Reis ${ }^{5}$; Andrei Pereira Pernambuco ${ }^{1,6}$
\end{abstract}

${ }^{1}$ Centro Universitário de Formiga (UNIFOR-MG). Departamento de Educação Física e Fisioterapia. Formiga, Minas Gerais, Brasil.

${ }^{2}$ Universidade Federal de Lavras - (UFLA). Departamento de Nutrição. Lavras, Minas Gerais, Brasil.

${ }^{3}$ Universidade de Franca - (UNIFRAN). Franca, São Paulo, Brasil.

${ }^{4}$ Centro Universitário de Varginha - (Unis MG). Varginha, Minas Gerais, Brasil.

${ }^{5}$ Universidad del Pacífico (UP). Departamento de Pós Graduação. Assunção, Paraguai.

${ }^{6}$ Universidade Federal de Minas Gerais- (UFMG). Instituto de Ciências Biológicas. Belo Horizonte, Minas Gerais, Brasil.

\section{Resumo}

Introdução: A gama de modificações fisiológicas que ocorrem durante e após a prática do exercício físico é ampla. Objetivo: Sendo assim, faz-se necessário uma compreensão mais ajustada e precisa das variáveis fisiológicas relacionadas ao exercício físico e nesse estudo a variabilidade da frequência cardíaca. Metodologia: Foram avaliadas 150 universitárias em um teste de cargas progressivas em esteira. O teste realizado foi o de carga progressiva em esteira pelo protocolo de Conconi até a fadiga medida pela percepção subjetiva de esforço. Resultados: Através da análise estatística detectou-se um $\mathrm{p}<0,05 \mathrm{com}$ diferenças estatísticas significativas entre 5 a $10 \mathrm{~km} / \mathrm{h}$ quando analisado o comportamento da variabilidade da frequência cardíaca. Apresentou-se também uma forte correlação negativa quando comparadas a variabilidade da frequência cardíaca e a percepção subjetiva de esforço com um valor de $\mathrm{P}<0.0001$ e $\mathrm{r}-0,9901$.O presente estudo mostra uma atividade simpática diminuindo consideravelmente a VCF nos primeiros estágios do teste de cargas progressivas. Mecanismos fisiológicos podem explicar isso parcialmente. Nas cargas iniciais do teste o sistema parassimpático começa a ser inibido devido à região bulbar e aumento concomitante do sistema simpático Conclusão: A pesquisa demonstrou que a VFC sofre adaptações e acabam por diminuir o intervalo R-R, quando os esforços físicos vão ficando mais intensos. Tais adaptações sofrem grande influência do sistema nervoso autônomo simpático.

Palavras-Chave: Variabilidade de Frequência Cardíaca. Percepção subjetiva de esforço. Teste de Cargas Progressivas. Escala de Borg.

Autor correspondente:

Alan Peloso Figueiredo

Endereço: Av. Bias Fortes, 82, Centro,

Varginha, $M G$, Brasil.

Recebido em: 06/10/2015

Telefone: +55 (35)3221-2707

Revisado em: 06/06/2016

Aceito em: 08/06/2016

E-mail alannf@terra.com.hr

Publicado em: 15/06/2016 


\section{Abstract}

Introduction: The behavior of the cardiac frequency variability in test with progressive loads the physiological modifications that occur during and after the physical activity are huge. Objective: Because of that, it's necessary to have a more accurate and precise comprehension of the physiological variables related to the exercise and in this study the heart rate variability. Methods: The test was applied in 150 female university students with progressive loads in a treadmill until the fatigue measured by a test of exertion subjective perception. After statistic analysis, it was a p<0,05 with significant statistic differences between $5-10 \mathrm{~km} / \mathrm{h}$ when analyzed the behavior of the cardiac frequency variability. Results: It was also noticed that there's a negative correlation between cardiac frequency variability and exertion subjective perception with $p<0,0001$ and $r$ of 0,9901. The present study shows that the sympathetic activity goes down considerably the cardiac frequency variability in the early stages of the test with progressive loads. Physiological mechanisms can explain it partially. In the early loads, the parasympathetic system starts to the inhibited by the bulb region and the concomitant increase of the sympathetic system. Conclusion: The research showed that the cardiac frequency variability suffers adaptations and end up decreasing the $R$ - $R$ interval when the physical exertion gets more intense. These adaptations suffer a huge influence by the sympathetic autonomic nervous system.

Keywords: Cardiac frequency variability, exertion subjective perception, test of progressive load, Borg scale.

\section{Introdução}

A gama de modificações fisiológicas que ocorrem durante e após a prática do exercício físico é ampla. Sendo assim, faz-se necessário uma compreensão mais ajustada e precisa das variáveis fisiológicas relacionadas ao exercício físico, tanto no que se refere ao seu comportamento, quanto em relação a sua aplicabilidade em prol da saúde dos envolvidos ${ }^{1}$.

De acordo com a literatura, a variabilidade da Frequência Cardíaca (VFC) é uma das variáveis mais utilizadas quando se pretende analisar a relação entre o Sistema Nervoso Autônomo (SNA) e o ritmo cardíaco. A variação a cada batimento obtida pelo intervalo R-R, que é caracterizado pelo tempo de intervalo de descargas elétricas do miocárdio, é então analisada pelo conjunto das frequências que compõem a variabilidade. Indivíduos sem cardiopatias apresentam variação fisiológica nos intervalos interbatimentos em fase com os ciclos respiratórios. A frequência cardíaca (FC) humana no repouso apresenta flutuações espontâneas que refletem a influência contínua do SNA no nodo sinoatrial. As oscilações devem ser corretamente quantificadas provendo assim um poderoso método de investigação do equilíbrio simpático-vagal no coração ${ }^{2,3}$.

Outra variável frequentemente utilizada em estudos médicos e/ou nas ciências do esporte é a percepção subjetiva de esforço (PSE). Esta pode ser definida como a tensão relativa que ocorre no sistema muscular, nervoso, cardiovascular e pulmonar, durante a atividade física, observada do ponto de vista da pessoa que está sendo avaliada ${ }^{4}$.
De acordo com o contexto acima, o propósito do presente estudo foi verificar o comportamento da variável VFC durante a prática de um teste físico com cargas progressivas e as PSE das participantes.

\section{Metodologia}

Tipo do estudo:

Trata-se de um estudo experimental, transversal e exploratório com abordagem quantitativa.

\section{Cuidados éticos}

A presente pesquisa somente foi iniciada após a aprovação do Comitê de Ética da Universidade Federal de Lavras. O protocolo de pesquisa foi inscrito na Plataforma Brasil sobre o número CAAE 17544213.4.0000.5148 no ano de 2013. Após o parecer favorável do Comitê de Ética por meio do parecer de número 370.220, foi solicitado aos interessados em participar da pesquisa que assinassem em duas vias o termo de consentimento livre e esclarecido (TCLE). Estas e demais considerações éticas se basearam na resolução 466/12 do Conselho Nacional de Saúde (CNS).

\section{Amostra}

A amostra foi composta por conveniência, as primeiras 150 universitárias do Curso de Educação Física do Centro Universitário de Formiga - MG que aceitaram participar da pesquisa e que atenderam aos critérios de inclusão e exclusão foram inscritas no estudo. Os critérios de inclusão foram: ter idade entre 18 e 25 anos, praticar atividade física regularmente (mínimo de quatro vezes por semana) e com características de alta intensidade tais como aulas de artes marciais, aulas de bike indoor, aulas de zumba 
ou semelhantes e atletas de modalidades esportivas. E os critérios de exclusão foram: histórico ou presença de enfermidade cardiovascular, presença de desconfortos na região torácica durante a atividade física, uso de medicamentos para cardiopatias, náuseas ou vômitos durante o teste, doença neuromusculoesquelética prévia.

\section{Instrumentação}

Os instrumentos para coleta de dados foram: monitor cardíaco Polar (Frequencímetro) RS800CX com as seguintes funções: teste de aptidão física ownindex® - Teste do comportamento de um atleta, baseado entre outros dados no registro da variabilidade da frequência cardíaca, e utilizando recursos do programa Polar Precision Performance, permitindo determinar situações de excesso de treinamento; gravação em intervalos - colhe amostras para criar um gráfico de Frequência Cardíaca. O gráfico ainda demonstra mais informações, tais como, Altitude, Velocidade, R-R, Cadência ( $1 \mathrm{~s}, 5 \mathrm{~s}, 15 \mathrm{~s}$ e $60 \mathrm{~s})$.

Escala de Borg de graduação de zero a 10, que mede a percepção Subjetiva de Esforço (PSE); esteira E720 Movement que permite velocidade de até 18 $\mathrm{km} /$ em uma inclinação de zero grau.

\section{Intervenção pré teste}

Os dados foram aferidos em horários prédeterminados, preferencialmente pela manhã, e as avaliadas foram orientadas a se alimentarem com café da manhã de seu costume, observando-se que deveriam consumir fontes de carboidratos a fim de evitar queda glicêmica. Tal alimentação foi feita duas horas antes da aplicação do teste.

\section{Coleta dos dados}

O frequencímetro foi colocado nas participantes ajustando os seguintes dados às características da participante: idade, peso, altura e sexo. Fixada a fita do frequencímetro na altura do peito, logo abaixo dos seios, esperou-se até que o mesmo conseguisse o sinal da frequência cardíaca. As voluntárias foram avaliadas em teste com cargas progressivas realizado em esteira ergométrica. O protocolo se iniciou com velocidade de $5,0 \mathrm{~km} / \mathrm{h}$, durante um minuto, elevando $1,0 \mathrm{~km} / \mathrm{h}$ adicionais a cada um minuto. Faltando dez segundos para uma nova carga, as voluntárias informaram, mediante uma Escala de Borg, sua PSE. A metodologia utilizada se baseou no teste de
Conconi que é amplamente usado em pesquisas com indivíduos saudáveis e também com doenças cardiovasculares ${ }^{5}$.

O medidor de frequência só foi retirado dois minutos após o término do teste. Após cada dia de coleta, os dados foram passados via função infravermelho para o software Polar Precision 2.7

\section{Estatística}

Os dados obtidos foram tabulados na planilha do software Microsoft Excel e em seguida foram calculadas as medidas de tendência central e de dispersão, média e desvio padrão de todas as variáveis no repouso e a cada aumento de $1 \mathrm{~km} / \mathrm{h}$ de acordo com o teste de Conconi. A seguir, para realizar a estatística, foi utilizado o programa GraphPadPrism v5.0 com nível de significância ajustado para $\alpha=0,05 \quad(\mathrm{p} \leq 0,05)$. Para avaliar a distribuição dos dados foi utilizado o teste de Kolmogorov Smirnov, para as comparações intragrupo foi utilizado o teste de Kruskal-Wallis. Já para a análise das correlações entre as variáveis foi utilizado o teste de Spearman. Para melhor visualização dos resultados, estes foram apresentados em gráficos.

\section{Resultados}

O grupo pesquisado foi constituído de 150 mulheres com idade entre 18 a 25 (média de 22,08 \pm 2,20 anos, altura média de166,07 $\pm 4,75 \mathrm{~cm}$, peso médio de $61,91 \pm 5,65 \mathrm{~kg}$ e IMC médio de $21,17 \pm 1,93$ $\mathrm{kg} / \mathrm{m} 2$.

A Figura 1 demonstra o comportamento da VFC durante o teste com cargas progressivas da velocidade $5 \mathrm{~km} / \mathrm{h}$ até a velocidade de $14 \mathrm{~km} / \mathrm{h}$ quando esta foi alcançada pelas participantes. Observa-se uma queda na VFC mais acentuada nas velocidades iniciais do teste entre $5 \mathrm{~km} / \mathrm{h}$ para $6 \mathrm{~km} / \mathrm{h}$ e assim consecutivamente até que se alcançou a velocidade de $10 \mathrm{~km} / \mathrm{h}$. Através da análise estatística detectou-se um $\mathrm{p}<0,05$ com diferenças estatísticas significativas. Entre 5 e $6 \mathrm{~km} / \mathrm{m}$ um $\mathrm{p}=0,015$, entre 6 e $7 \mathrm{~km} / \mathrm{h}$ $\mathrm{p}=0,017$, entre 7 e $8 \mathrm{~km} / \mathrm{h} \mathrm{p}=0,016$, entre 8 e $9 \mathrm{~km} / \mathrm{h}$ $\mathrm{p}=0,016$ e entre 9 e $10 \mathrm{~km} / \mathrm{h} \mathrm{p}=0,01$. Os incrementos de carga a partir de $10 \mathrm{~km} / \mathrm{h}$ não resultaram em significância estatística quando comparados com a carga imediatamente anterior. 


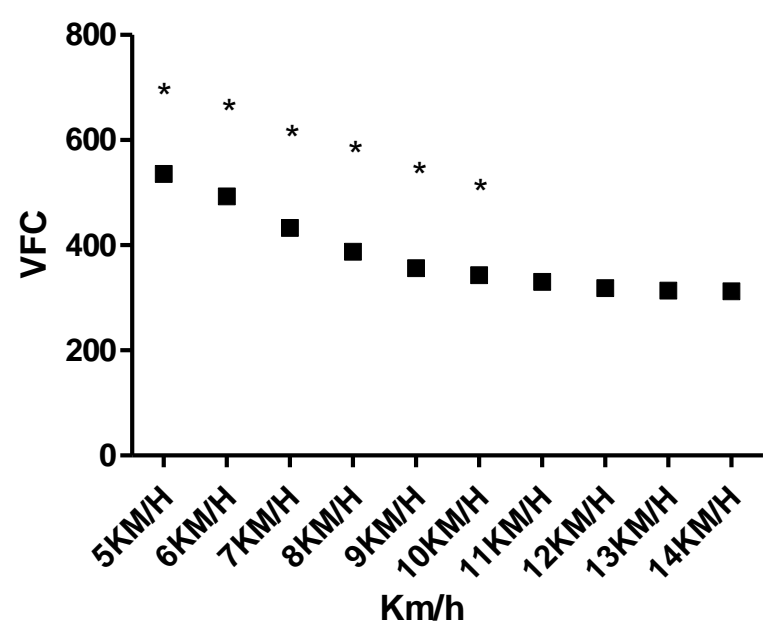

Figura 1- Comportamento da VFC . * demonstra que a VFC foi estatisticamente diferente quando se comparou duas cargas consecutivas.

Figura 2 demonstra uma forte correlação negativa e significativa entre as médias de PSE obtidas com o uso da Escala de Borg nas diferentes cargas oferecidas às participantes e os valores da VFC nos momentos correspondentes. Valor de $\mathrm{p}$ $<0.0001$ e r $-0,9901$.

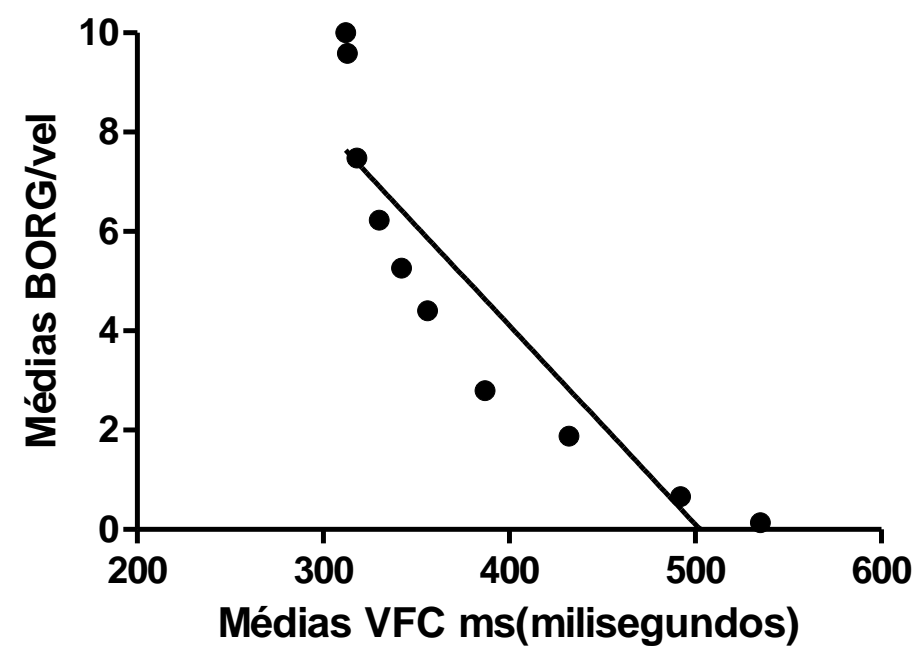

Figura 2- Correlação das médias da Escala de Borg com as médias da VFC em diferentes momentos do estudo.

\section{Discussão}

Os resultados do presente estudo sugerem a diminuição da atividade parassimpática nos primeiros estágios de um teste de cargas progressivas, evidenciada sobretudo, pela redução da VFC ao longo do teste. Os resultados do presente estudo corroboram os achados de Vieira e Felix ${ }^{6}$ que evidenciaram este tipo de padrão também em idosos. Além do mais, os resultados aqui demonstrados evidenciaram que a redução linear da VFC aconteceu principalmente até a velocidade de $10 \mathrm{Km} / \mathrm{h}$ e que após a velocidade de $11 \mathrm{Km} / \mathrm{h}$ os valores da VFC se estabilizaram. De acordo com Caruso e colaboradores $^{7}$, quanto mais próximo da frequência cardíaca máxima menor será a diminuição da VFC, pois mesmo com o efeito do sistema simpático não há condições fisiológicas cardíacas possíveis para isso. Nesse sentido o presente estudo corrobora com o estudo dos autores.

Alguns mecanismos fisiológicos podem explicar tal fato, mesmo que parcialmente. Nas cargas iniciais do teste o sistema parassimpático começa a ser inibido devido à ativação da região bulbar e aumento concomitante do sistema simpático. A ativação simpática e inibição parassimpática sobre o coração resultam no aumento da frequência cardíaca e no aumento da força de contratilidade do miocárdio, dentre outras. Os dados do presente estudo, novamente corroboraram com os achados de outro estudo realizado com homens e mulheres jovens ${ }^{8}$. 
Quando se posicionou a correlação das médias da escala de Borg com as da VFC demonstrou-se uma correlação significativa inversamente proporcional, ou seja, quanto maior a carga de PSE assumida pelas avaliadas, menor era o intervalo da variabilidade cardíaca.

Os fatores iniciais dos testes progressivos parecem assumir maior amplitude de ajustes que os fatores a longo prazo. Entre eles pode-se citar a influência das catecolaminas sobre o coração aumentando assim a FC e consequentemente diminuindo a VFC. Estes e outros ajustes se fazem necessários para suprir a crescente demanda sanguínea dos músculos decorrentes da prática de atividade física, contudo, quando cargas adicionais são impostas, os receptores, por já estarem saturados de catecolaminas, já não mais respondem prontamente à diminuição da $\mathrm{VFC}^{2,8}$.

\section{Conclusão}

A pesquisa demonstrou que a VFC sofre adaptações e acaba por diminuir o intervalo R-R, quando os esforços físicos se tornam mais intensos. Tais adaptações sofrem grande influência do sistema nervoso autônomo simpático que torna-se cada vez mais ativo durante os exercícios físicos, contrapondo assim a ação inibitória parassimpática sobre o coração.

O aumento da atividade simpática foi maior nos estágios iniciais do teste e após determinado período a redução não foi significativa, o que se deve, pelo menos em parte, pelo fato de os receptores de catecolaminas estarem saturados. Além do mais, há forte correlação negativa encontrada entre a PSE e a VFC, o que demonstra que a PSE é um bom marcador do comportamento da VFC e, portanto, pode ser utilizada para predizer o comportamento da VFC durante os testes de esforço.

Sugere-se que novos estudos sejam realizados com objetivos semelhantes aos deste, a fim de verificar o comportamento de outras variáveis durante os testes de esforço, para que deste modo os profissionais da área de saúde tenham cada vez mais parâmetros confiáveis para prescrever e implementar exercícios físicos de alta intensidade.

\section{Declaração de conflitos de interesses}

Os autores do artigo afirmam não haver nenhuma situação de conflito de interesse.

\section{Referências}

1. DE SOUZA, KRISTOPHER MENDES et al. Maximal lactate steady state estimated by differen tmethods of anaerobic threshold. Brazilian Journal of Kinanthropometry and Human Performance, [S.1.], v. 14, n. 3, p. 264-275, apr. 2012.
2. BRANDÃO GS, et al. Análise da variabilidade da frequência cardíaca na mensuração da atividade do sistema nervoso autônomo: MTP \& Rehab Journal 2014, 12:630655.

3. VANDERLEI LCM, et al. Noções básicas de variabilidade da frequência cardíaca e sua aplicabilidade clínica. Bras Cir Cardiovasc 2009; 24(2): 205-21.

4. FINNKELSTEIN I, et al. Comparação do índice de esforço percebido e consumo de oxigênio em exercício em cicloergômetro entre gestantes e não-gestantes e entre o exercício aquático e terrestre. RevBrasMed Esporte 2012; 18(1): 13-16.

4. GRAZZI G, MYERS J, BERNARDI E, TERRANOVA F, GROSSI G, CODECA L, et al. Association between $\mathrm{VO}_{2}$ peak estimated by a $1-\mathrm{km}$ treadmill walk and mortality. A 10-year follow-up study in patients with cardiovascular disease.Int J Cardiol 2014; 173(2): 248-52

5. VIEIRA S, FELIX ACS, QUITERIO RJ. Variabilidade da frequência cardíaca e carga máxima atingida no teste de esforço físico dinâmico em homens idosos. Ver Bras Med Esporte 2012; 18(6): 377-380.

6. CARUSO FCR, et al. Determinação do limiar anaeróbio pela variabilidade da frequência cardíaca de pacientes com DPOC durante exercício em cicloergômetro. Fisioter Mov 2012; 25(4): 117-128.

7. COUTO PG, et al. Pontos de transição da frequência cardíaca em teste progressivo máximo. Motriz 2013; 19(2): 261-268. 